\title{
Apoptosis Detection for Non-adherent Cells in Time-lapse Phase Contrast Microscopy
}

\author{
Seungil Huh and Takeo Kanade \\ Lane Center for Computational Biology and Robotics Institute \\ Carnegie Mellon University \\ \{seungilh,tk\}@cs.cmu.edu
}

\begin{abstract}
This paper proposes a vision-based method for detecting apoptosis (programmed cell death), which is essential for non-perturbative monitoring of cell expansion. Our method targets non-adherent cells, which float or are suspended freely in the culture medium - in contrast to adherent cells, which are attached to a petri dish. The method first detects cell regions and tracks them over time, resulting in the construction of cell tracklets. For each of the tracklets, visual properties of the cell are then examined to know whether and when the tracklet shows a transition from a live cell to a dead cell, in order to determine the occurrence and timing of a cell death event. For the validation, a transductive learning framework is adopted to utilize unlabeled data in addition to labeled data. Our method achieved promising performance in the experiments with hematopoietic stem cell (HSC) populations, which are currently in clinical use for rescuing hematopoietic function during bone marrow transplants.
\end{abstract}

\section{Introduction}

One major research topic in stem cell engineering is to study cell proliferation to facilitate the expansion of stem cells with minimal cell death. In order to understand the mechanism of cell expansion, researchers often quantitatively analyze cell growth in response to various engineered microenvironments. Accordingly, automated vision systems for monitoring cell birth and death, particularly adopting time-lapse phase contrast microscopy which is a popular and non-destructive microscopy modality, are of great interest in the community.

Recent studies have demonstrated great success at detecting cell birth, or mitosis, in time-lapse phase contrast microscopy (We refer to [1] for the review of mitosis detection algorithms.). More recently a method for apoptosis (programmed cell death) detection in time-lapse phase contrast microscopy was proposed for adherent cells and showed its success on $\mathrm{C} 2 \mathrm{C} 12$ myoblastic stem cell data [2]. However, unfortunately, this method cannot be applied to non-adherent cells because the detection relies on visual changes in cell morphology and image intensity during apoptosis, which are not observed among non-adherent cells. 
In this paper, we propose a method for detecting apoptosi 1 that is particularly devised for non-adherent cells. Since non-adherent cells do not show distinctive shape or brightness change during apoptosis, rather than focusing on the apoptotic process occurring in a short time period, our method investigates a long-term transition from a live cell to a dead cell in cell tracklets generated as a result of cell tracking. Each tracklet is examined using a statistical model in order to determine the occurrence and timing of a death event in the tracklet. For the validation, we adopt a transductive support vector machine (TSVM) in order to use unlabeled data as well as labeled data.

The proposed method was tested on 14 hematopoietic stem cell (HSC) populations and achieved encouraging performance. Since HSCs are currently in clinical use for rescuing hematopoietic function during bone marrow transplants, but the HSC therapy is still limited by the low cell dose available, we expect that this work would contribute to increasing knowledge on HSC expansion.

\section{Previous Work}

In biology, apoptosis detection is currently performed by measuring the levels and activity of apoptotic molecules using staining methods with a variety of assays. However, such a method is not appropriate for a long-term monitoring of intact cells since phototoxicity due to the staining chemicals may affect cell behavior. Vision-based methods adopting non-fluorescent microscopy often implicitly detected apoptosis during cell tracking by regarding track termination as cell death, albeit with poor performance. Recently, 2] proposed a vision-based apoptosis detection method for adherent cell populations and showed its success in $\mathrm{C} 2 \mathrm{C} 12$ stem cell populations. To the best of our knowledge, there has not been vision-based work for detecting apoptosis in a non-adherent cell population.

\section{Death Event of Non-adherent Cells}

The objective of apoptosis detection is to know the timing (frame id) and location ( $\mathrm{x}$ and $\mathrm{y}$ positions) of each death event, which we define as the completion of apoptosis. In this section, we describe visual characteristics of apoptosis and death events of non-adherent cells to provide a better understanding of the task.

Apoptosis of non-adherent cells involves a series of events: membrane blebbing, DNA degradation, and the formation of apoptotic bodies. As blebs are formed and the contents of cells spill out, cells undergoing apoptosis are often visually characterized by irregular shape and outline, as shown in Fig. 11(a,b). Sometimes (a few percentage of the total in our experiments), cells are dying while becoming weaker without shape change, as shown in Fig. 11 $(c, d)$. Since there are a number of apoptotic stimulus sources, the cell dying process is not uniquely characterized, but shows various visual presentations and takes different amount of time from a few

${ }^{1}$ Following the previous work [2, we detect apoptosis among different types of cell deaths because it is the most common and interesting in the community. 

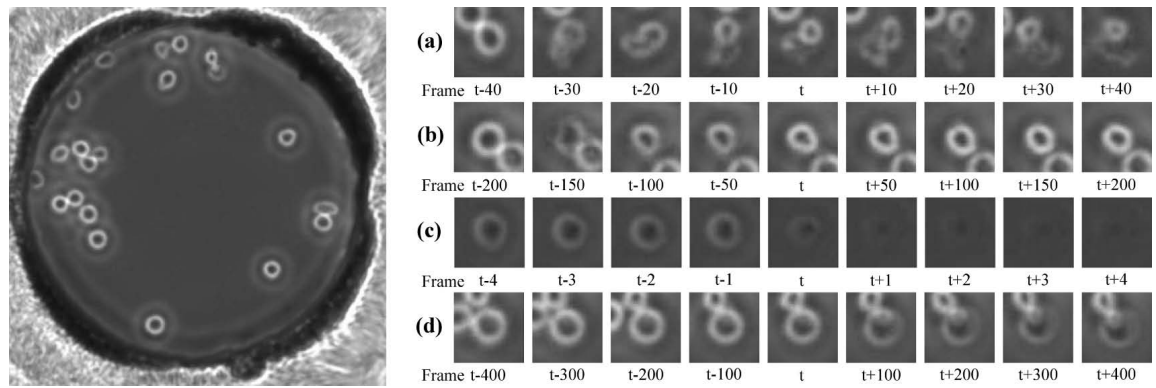

Fig. 1. Sample image frame (left) and patch sequences containing a death event which is manually annotated with the assistance of a biologist and aligned at frame $t$ (right)

minutes to several hours. After a death event, cell fragments get sticky and form a cluster, often becoming a circular shape again, the size of which is commonly smaller than it was alive, as shown in Fig. 1(b). After a death event, or apoptosis is completed, the cell is dead and thus does not move without external force.

A major difficulty of cell death event detection lies in the fact that live and dead cells are often not distinguishable from each other based on short-term observation due to their similar appearance and behavior. Specifically, live cells also occasionally show irregular shape and outline as they move and elongate; live cells sometimes appear dark when they are in contact with the microwell boundary; dead cells often show a circular shape and bright boundary after apoptotic bodies stick together; and live cells often show minimal movement for a long time, while dead cells occasionally vibrate along with the culture medium or move as being pushed by their neighboring live cells.

\section{Algorithm}

Since apoptosis of non-adherent cells is not visually distinctive with short-term observation, we detect a death event by tracing and examining the change of visual properties of the cell in a long time period. Specifically, we build tracklets (fragments of a cell trajectory that are likely to track the same cell without any error) by cell tracking and examine whether each tracklet shows a transition from a live cell to a dead cell to find a death event. For the validation, we adopt transductive modeling that uses unlabeled information in addition to labeled information, in order to reduce efforts of manual labeling as well as improve detection accuracy. Based on these ideas, we propose a method consisting of three steps: tracklet generation, feature extraction, and tracklet validation.

(1) Tracklet Generation: In order to construct tracklets, we track cells based on frame-by-frame association and detect mitosis by examining cell size change over time, using the method introduced in [3], as illustrated in Fig. 2, To make tracklets reliably track the same cell and thus be less prone to error, we adjust the tracking parameters so that a new tracklet may initiate rather than an association being conducted, when the confidence of frame-by-frame association is not high enough. Fig. 3 shows examples of cell tracklets. 
(a)

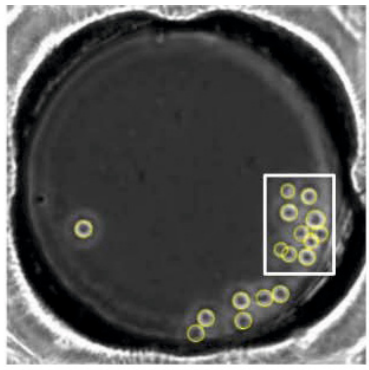

Frame t (b)

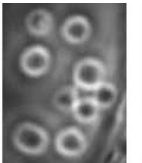

(c)

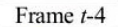

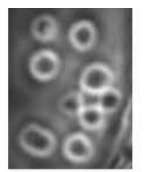

Frame $t-3$

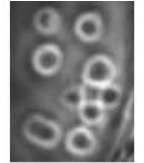

Frame $t-2$

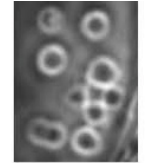

Frame $t-1$

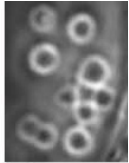

Frame $t$

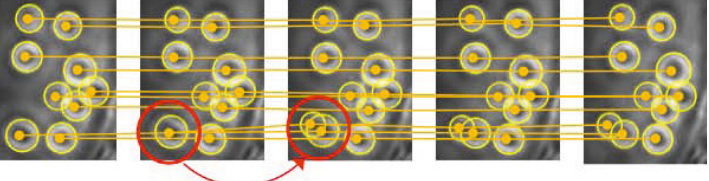

Mitosis

Fig. 2. Cell tracking and mitosis detection using the method in 3. (a) A sample image frame where cells are detected using a set of ring filters. (b) Original image sequence of the boxed area extracted from consecutive frames. (c) Cell tracking result marked by yellow dots and lines, and mitosis detection result by red circles and arrow.
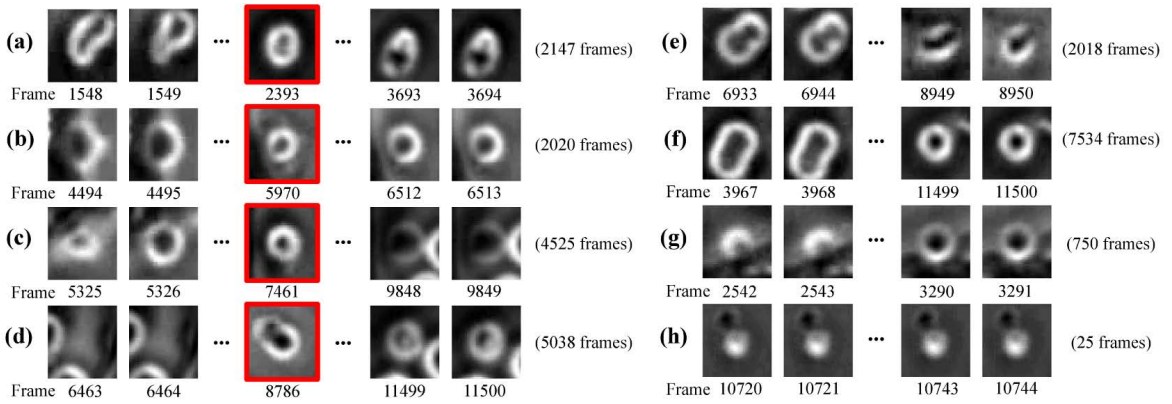

Fig. 3. Sample tracklets that (a-d) contain a death event and (e-h) do not contain any death event. The patches enclosed by a red box show a death event annotated by human. Tracklets consist of a varying number of patches, from tens to thousands.

It is worth mentioning that even tracklets may contain some errors. The errors can be compensated for to some extent as we more concern about the overall pattern change than the details of a small number of patches.

(2) Feature Extraction: Based on the discussion in Section 3, in order to capture a long-term cell property change, we extract the following high-level properties on cell appearance and movement:

- cell radius

- cell roundness

- displacement from the previous position

- whether being in contact with another cell

The first two features are computed based on the cell region detection result and the other features based on the cell tracking result. The last feature is used to measure the possibility of a cell's shape or movement being affected by neighboring cells. 
(a)

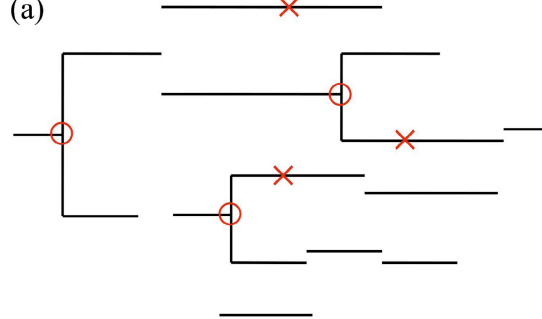

(b)

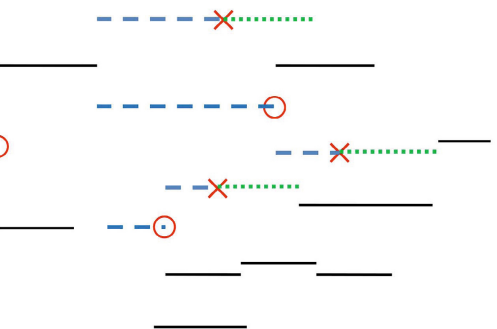

$\times$ Annotated death event $\bigcirc$ Detected mitosis $\quad-\quad$ Live cell tracklet $\quad \cdots$... Dead cell tracklet $\quad$ Unknown tracklet

Fig. 4. Conversion from labels on death events to those on tracklets (a) tracking results with manually annotated death events and automatically detected mitosis (b) tracklets categorized into three types: tracklet of a live cell, a dead cell, and a cell unknown whether it is live or dead. A tracklet containing a death event is divided into two tracklets and the first and second pieces are labeled as tracklets of a live and a dead cell, respectively. The tracklet of a mother cell is labeled as the tracklet of a live cell.

As a result of feature extraction from each tracklet, we obtain a time series of feature vectors where the length of the time series is the same as the number of patches in the tracklet and each feature vector consists of the four cell properties. To obtain the same size of features from all tracklets, we resize each time series into a certain length $S$ by averaging features among consecutive patches or interpolating features of between-patches. The feature vectors of resized time series are then concatenated into one feature vector, which is the feature of the tracklet consisting of $4 \mathrm{~S}$ elements. In our experiments, $S$ was set to be 100 after testing a set of values, $\{10,30,50,100,200,300\}$, based on the apoptosis detection accuracy on a validation set. The accuracy is not sensitive to $S$ as long as it is within a reasonable range; in our experiments, the choice of either 50 or 200 for $S$ results in only less than $1 \%$ performance drop.

(3) Tracklet Validation: A way to validate tracklets would be using a supervised learning-based method that models the occurrence and timing of death events. Instead of this typical approach, i.e., explicitly modeling death events in tracklets in a supervised learning framework, we model tracklets of live cell and dead cells, the transition of which indicates a death event, in a semi-supervised learning framework. To do so, we need to convert labels on death events into labels on tracklets as illustrated in Fig. 4, since the manual annotation is given on each death event, a specified time and location, rather than each tracklet. The procedure of the labeling conversion is as follow. First, since a death event by definition means a transition from a live cell to a dead cell, we divide the tracklets containing a manually annotated death event into two tracklets and label the first and second pieces as tracklets of a live and a dead cell, respectively. Second, because a dead cell cannot undergo mitosis, we label the tracklet of a mother cell as the tracklet of a live cell. The remaining tracklets are assigned as tracklets of a cell unknown whether it is alive or dead.

In order to utilize the unlabeled information in addition to the labeled information, we adopt a transductive support vector machine (TSVM) 4]. TSVMs 
additionally consider unlabeled data; while learning a maximum margin classifier with labeled samples like (inductive) SVMs, TSVMs simultaneously force the margin to be as much far away as possible from unlabeled samples. More formally, the optimization problem of TSVM is as follows: given a set of $L$ labeled samples $\mathcal{L}=\left\{\left(\mathbf{x}_{1}, y_{1}\right), \cdots,\left(\mathbf{x}_{L}, y_{L}\right)\right\}, \mathbf{x} \in \mathbb{R}^{4 S}, y \in\{1,-1\}$ and a set of $U$ unlabeled samples $\mathcal{U}=\left\{\mathbf{x}_{L+1}, \cdots, \mathbf{x}_{L+U}\right\}$, TSVM minimizes

$$
\frac{1}{2}\|\omega\|^{2}+C^{(L)} \sum_{i=1}^{L} \xi_{i}+C^{(U)} \sum_{i=L+1}^{L+U} \xi_{i}
$$

subject to

$$
\begin{array}{rll}
y_{i}\left(\omega \cdot \mathbf{x}_{i}+b\right) \geq 1-\xi_{i}, & \xi_{i}>0 & \text { for } i=1, \cdots, L \\
\left|\omega \cdot \mathbf{x}_{i}+b\right| \geq 1-\xi_{i}, & \xi_{i}>0 & \text { for } i=L+1, \cdots, L+U
\end{array}
$$

where $C^{(L)}$ and $C^{(U)}$ are SVM parameters for labeled and unlabeled samples, respectively, and $\{\xi\}$ is a set of slack variables for soft margin SVMs. This is a combinatorial optimization problem and thus finding the optimal solution is intractable for a large dataset. Instead, to obtain an approximate solution, we adopt a local search-based method that begins with an inductive SVM solution and keeps updating the solution by increasing the influence of the test examples. We refer to [4] for more detailed procedure of the TSVM optimization.

In the testing phase, given a time series of features of a new tracklet, we divide the time series into two pieces at various time points uniformly distributed on the time series and examine whether the first and second pieces are tracklets of a live cell and a dead cell respectively, using the trained transductive SVM. If there is a case that both are likely to be true, the tracklet is determined to contain a death event and its timing is set corresponding to the best partition. Otherwise, the tracklet is considered not to contain a death event.

\section{Experiments}

After describing the experimental setup, we empirically show the effectiveness of the proposed method against alternative methods with discussions.

(1) Data and Annotation: The proposed method was tested on 14 timelapse phase contrast microscopy images, each of which consists of 11520 frames. For more detailed information on image acquisition, see [3]. The data and annotation are available in the first author's home page (www.cs.cmu.edu/ seungilh).

On the time-lapse images, we manually annotated death events with the assistance of a biologist. For the annotation, we first detected dead cells and investigated their tracks in reverse direction to precisely identify the timing of the apoptosis completion based on cell appearance change and movement. For each death event, we marked the center of the dead cell. As a result, we annotated 52 death events in total in the 14 time-lapse microscopy images.

(2) Evaluation and Comparison: We define a true positive as the case that a tracklet containing a death event is correctly identified. To minimize the 


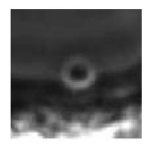

Frame 9800

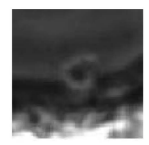

Frame 9900

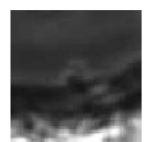

Frame 9954 (missed)

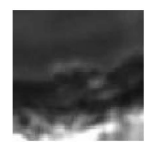

Frame 10100

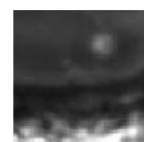

Frame 11260 (death event)

Fig. 5. Death event undetected in the cell region detection step. The dying cell was not captured because it is not bright due to the vicinity to the well boundary.

sampling bias, we performed a leave-one-sequence-out test; i.e., we used one sequence as a test set in turn and the others as a training set. Summing up all the results, we computed precision, recall, and F-measure. If one tracklet contains more than one death event due to erroneous tracking, all the death events except one in the tracklet were considered undetected, or false negatives.

We compared the proposed approach against a typical sliding window scheme as a baseline method, which examines a fixed length of consecutive patches in every possible location to determine whether it contains a death event. Positive samples were extracted from every tracklet containing a death event, specifically from the window at the center of which the death event is located. Negative samples were extracted from the windows randomly located in the tracklets not containing a death event. Note that a sliding window scheme models death events, not tracklets of live and dead cells. For the validation of each sample, we applied (inductive) SVM since there is no unlabeled data.

We also compared our transductive SVM model with inductive SVM and Hidden Conditional Random Field (HCRF) [5], which have been popularly used for event detection in time-lapse images. For the comparison, features were extracted in the same way as the proposed method and only the statical model was replaced. Note that these models are not able to utilize unlabeled information.

(3) Results and Discussions: In the candidate detection step, total 1056 tracklets were constructed. Tracklets are much more than the branches in the actual lineage trees because new tracklets are initiated whenever cell region association is not confident. These tracklets captured 49 out of 52 death events. Two death events were missed because the dying cells were not localized in the cell region detection step as they stick to the microwell boundary and thus do not show a bright boundary as shown in Fig. 5. The other death event was considered missed as the tracklet containing it also contains another death event.

The proposed method achieved $79.2 \%$ precision and $73.1 \%$ recall. In terms of F-measure, it achieved $76.0 \%$ accuracy. The proposed method outperforms a typical sliding window method, which is the baseline method, by $11.6 \%$ in terms of F-measure as reported in Table 1, While the proposed method captures a long-term transition from a live cell to a dead cell, a sliding window method refers to only local information in the window, and thus it is not effective to detect a death event, which does not involve a short-term visual hallmark nor evident visual change between prior to and after the event. 
Table 1. Death event detection performance comparison (*: proposed method)

\begin{tabular}{|c|c|c|c|c|}
\hline Features extraction from & Validation with & Precision & Recall & F-measure \\
\hline Whole tracklet & TSVM & 0.792 & 0.731 & $0.760^{*}$ \\
\hline Sliding window & SVM & 0.824 & 0.528 & 0.644 \\
\hline Whole tracklet & SVM & 0.805 & 0.635 & 0.710 \\
\hline Whole tracklet & HCRF & 0.449 & 0.585 & 0.508 \\
\hline
\end{tabular}

The semi-supervised approach with TSVM outperforms (inductive) SVM or HCRF as demonstrated in Table 1. Unlike SVM or HCRF, TSVM takes into account the distribution of unlabeled data, the use of which is not only helpful to improve the accuracy, but also desirable because manual labeling is timeconsuming and labor-intensive in bioimage analysis.

For the statistical test, we repeated an experiment 20 times with randomly selected half of sequences as a training set and the other half as a testing set. Student $t$-test with $1 \%$ confidence level reveals that the outperformance of our method over the others is statistically significant.

\section{Conclusion}

We propose a vision-based method of apoptosis detection for non-adherent cells in time-lapse phase contrast microscopy, which is an essential component for nonperturbative monitoring of cell expansion. Since apoptosis of non-adherent cells do not show a short-term distinctive visual hallmark, we search for a long-term transition from a live cell to a dead cell using cell tracking and semi-supervised learning. Experiments on HSC data demonstrate promising results.

In addition to enhancing image quality, the use of more sophisticated low level features from image patches and time series classification without matching the length of tracklets might help improve the apoptosis detection accuracy. We leave the experimental validation as future work.

\section{References}

1. Huh, S., Ker, D.F.E., Bise, R., Chen, M., Kanade, T.: Automated Mitosis Detection of Stem Cell Populations in Phase-Contrast Microscopy Images. IEEE Trans. Med. Imaging 30(3), 586-596 (2011)

2. Huh, S., Ker, D.F.E., Su, H., Kanade, T.: Apoptosis Detection for Adherent Cell Populations in Time-Lapse Phase-Contrast Microscopy Images. In: Ayache, N., Delingette, H., Golland, P., Mori, K. (eds.) MICCAI 2012, Part I. LNCS, vol. 7510, pp. 331-339. Springer, Heidelberg (2012)

3. Huh, S., Eom, S., Ker, D.F.E., Weiss, L.E., Kanade, T.: Mitosis Detection of Hematopoietic Stem Cell Populations in Time-lapse Phase-contrast Microscopy Images. In: Proc. IEEE ISBI, pp. 390-393 (2012)

4. Joachims, T.: Transductive Inference for Text Classification using Support Vector Machines. In: Proc. ICML, pp. 200-209 (1999)

5. Quattoni, A., Wang, S., Morency, L., Collins, M., Darrell, T.: Hidden conditional random fields. IEEE Trans. Pattern Anal. Mach. Intell. 29(10), 1848-1853 (2007) 